\title{
Single Dose Propranolol Does Not Affect Physiologic or Emotional Reactivity to Smoking Cues
}

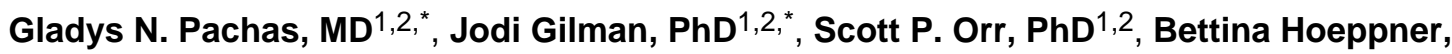 \\ PhD $^{1,2}$, Sara V. Carlini ${ }^{1}$, Tsafrir Loebl, MD ${ }^{1}$, Johanna Nino, MD ${ }^{1}$, Roger K. Pitman, MD ${ }^{1,2}$, \\ and A. Eden Evins, MD, MPH ${ }^{1,2}$ \\ ${ }^{1}$ Department of Psychiatry, Massachusetts General Hospital, Boston Massachusetts \\ ${ }^{2}$ Harvard Medical School, Boston Massachusetts
}

\section{Abstract}

Background-Smoking cue exposure reactivates salient smoking-related memories, triggering craving to smoke, a phenomenon associated with maintenance of smoking behavior and relapse after periods of abstinence. Acute $\beta$-adrenergic blockade with propranolol reduces physiologic reactivity during subsequent recollection of traumatic events by inhibiting reconsolidation of reactivated memories in a process called memory reconsolidation blockade.

Objective-To determine whether a single dose of propranolol prior to retrieval of smokingrelated memories reduces subsequent physiologic reactivity to personally salient smoking imagery scripts in current smokers.

Methods-Fifty-four overnight-abstinent, adult smokers received single dose propranolol or placebo prior to reactivation of smoking-related memories in a randomized, double-blind, placebocontrolled trial and resumed smoking afterward. One week later, skin conductance (SC), heart rate (HR) left corrugator electromyogram (EMG), self-reported emotional state and craving were assessed following script driven imagery with neutral and personalized smoking-related scripts.

Results-Smoking scripts were associated with increased physiologic activation (SC, HR, EMG), craving and negative emotional state compared with neutral scripts. Propranolol did not moderate the effect of script type on any outcome.

Conclusion-Personalized smoking script-driven imagery robustly increased physiologic activation, negative emotional state and craving, and a single dose of propranolol prior to memory reactivation did not moderate this effect.

Correspondence to: A. Eden Evins, MD, MPH, Director, Massachusetts General Hospital, Center for Addiction Medicine, 60 Staniford Street Boston, MA 02114 a_eden_evins@hms.harvard.edu.

*Contributed equally to the manuscript.

Presented in part at the 2012 Society for Research on Nicotine and Tobacco, Houston TX

Declaration of Conflicting Interests: Dr. Evins has received research grant support from Pfizer, Envivo Pharmaceuticals, Janssen, GSK, and has consulted to Pfizer and Boehringer Ingelheim. Drs. Pachas, Gilman, Orr, Pitman, Loebl, Nino-Gomez, and Hoeppner and Ms. Carlini have no conflicts to disclose. 


\section{Keywords}

propranolol; smoking; memory reconsolidation blockade; cue; reactivity; craving; affect; electrophysiology

\section{INTRODUCTION}

Tobacco smoking is the leading cause of preventable mortality in developed countries. The mechanisms underlying maintenance of smoking behavior and relapse to smoking after initial abstinence are thought to be multifactorial, involving affective, motivational, and cognitive processes, influenced by negative affect, perceived stress, and exposure to environmental cues previously associated with smoking, as well as the smoker's emotional reaction to such factors and expectation that smoking will ameliorate associated negative affect (Chiamulera et al. 1990; Shiffman 2005; Weiss 2005). During episodes of addictive drug use, the brain forms associations between neutral environmental cues and the rewarding effects of the drug in a process by which addictive substances stimulate phasic dopamine release, which increases the salience of the memory of the drug experience and associated factors. These cues alone can later trigger craving when reencountered, even after substantial periods of abstinence (Childress et al. 1988; Fricks-Gleason and Marshall 2008). In smokers, cue-induced craving is accompanied by negative affect (Drobes and Tiffany 1997) and physiologic activation that includes increased heart rate (Abrams et al. 1988; Abrams et al. 1987), blood pressure (Rickard-Figueroa and Zeichner 1985), and skin conductance (Abrams et al. 1988). Imagery-based cue-reactivity studies have demonstrated that detailed descriptions of personal smoking-related experiences, personalized smoking scripts, elicit strong craving responses accompanied by increased physiologic reactivity and negative affect (Conklin and Tiffany 2001; Drobes and Tiffany 1997; Tiffany and Hakenewerth 1991).

Cocaine-dependent individuals demonstrate greater glucose metabolism in brain regions involved in cognitive and affective aspects of memory consolidation after exposure to cocaine-related images, suggesting that environmental cues can become interconnected with cocaine craving by means of a distributed neural network that integrates emotional and cognitive aspects of memory (Grant et al. 1996). Once consolidated, drug-related memories are thought to be relatively stable and resistant to disruption (Alberini 2005). Hence, therapies intended to block consolidation of a drug-related memory would have to be administered within a few hours of initial drug use, making this approach impractical. A more promising therapeutic target is drug-associated memory retrieval and reconsolidation. Animal studies have reported that during memory retrieval, consolidated memories become labile and then must be actively reconsolidated in order to persist (Nader et al. 2000). If this process of memory reconsolidation could be disrupted, the learned association between the drug and its associated cues could potentially be broken, rendering formerly drug-related cues less salient and less liable to trigger craving and subsequent drug use. This process of impeding the reconsolidation of reactivated stable memories has been termed memory reconsolidation blockade and has been demonstrated in species ranging from snails to humans (recently reviewed in (Auber et al. 2013); performing appetitive, aversive, and 
neutral tasks; using approaches including systemic or localized drug administration, gene manipulation, and interference by new learning (Alberini 2005; Nader 2003). Memory reconsolidation blockade is distinct from extinction in that it can occur even when a reinforced trial is used to reactivate the memory (Bozon et al. 2003; Duvarci and Nader 2004); it does not show renewal after contextual shift (Duvarci and Nader 2004); it has a distinct neurochemical signature (Suzuki et al. 2004); and it is thought to directly target the original drug-associated memory, whereas extinction attempts to inhibit the expression of the consolidated memory through new inhibitory learning.

Memory reconsolidation can be disrupted by propranolol, a $\beta$-adrenergic receptor agonist (Przybyslawski et al. 1999). When administered following memory reactivation, propranolol has been shown to reduce inhibitory avoidance (Duvarci and Nader 2004; Przybyslawski et al. 1999), auditory fear conditioning (Debiec and Ledoux 2004), cocaine- (Bernardi et al. 2006; Fricks-Gleason and Marshall 2008) and morphine-conditioned (Robinson and Franklin 2007) place preference, and context-induced sucrose seeking (Diergaarde et al. 2006) in animals. In the first trial of memory reconsolidation blockade in humans, in a clinical sample, single dose propranolol reduced physiologic responding during imagery of a prior traumatic event when administered after reactivation of the traumatic memory (Brunet et al. 2008). Subsequent studies in humans have shown promise for the $\beta$-blocker, propranolol, to block the consolidation/reconsolidation of emotional memories in healthy adults. These include open label studies of single or repeated dosing of propranolol (Brunet et al. 2011; Poundja et al. 2012) and a controlled trial (Kindt et al. 2009), (reviewed in Lonergan et al. 2013). While memory reconsolidation blockade has been shown to abolish the reinforcing properties of drug-related cues (Lee et al. 2005) and disrupt the reinforcing properties of an appetitively reinforced conditioned stimulus (Milton et al. 2008b), translation of these findings to humans has been difficult. However, in a promising recent trial, propranolol, compared to placebo, reduced cocaine-cue related craving and cardiovascular reactivity in people with cocaine dependence 24 hours after dosing, though there was no lasting effect one week later (Saladin et al., 2013).

This study was based on the hypothesis that if there is a period of memory lability between reactivation and reconsolidation of highly salient, drug-related memories, and that reconsolidation of drug-related memories can be disrupted through reconsolidation blockade, a therapy administered at the time of memory reactivation could reduce the salience of drug-related memories, and reduce drug associated cue reactivity in humans. To test this hypothesis, we administered a single dose of propranolol or placebo to overnight abstinent smokers immediately prior to retrieval of smoking-associated memories and assessed for drug effect on smoking cue-associated physiologic reactivity, negative affect and craving, elicited with personalized imagery-based smoking cue scripts one week later.

\section{METHOD AND MATERIALS}

\section{Study Design}

Participants were enrolled from May 2008 to March 2010 at the Massachusetts General Hospital in compliance with the Declaration of Helsinki, the U.S. Food and Drug Administration guidelines, and the International Conference on Harmonization Good 
Clinical Practices Guidelines. The Partners Human Subjects Committee approved the study protocol, and participants provided written informed consent to participate following full description of study procedures prior to participation.

\section{Study Population and Recruitment}

Participants were otherwise healthy, nicotine dependent smokers, aged 18-65, who reported smoking 10 or more cigarettes per day for the prior 3 months and had expired CO $>10 \mathrm{ppm}$ (Bedfont Smokerlyzer II, Bedfont, Kent, England). Participants were excluded if they had systolic blood pressure $<100 \mathrm{~mm} \mathrm{Hg}$ at screening; a medical contraindication to propranolol administration or concurrent use of $\beta$-blockers, antiarrhythmic agents, calcium channel blockers, varenicline, bupropion or nicotine replacement therapy; met DSM-IV criteria for a substance use disorder other than nicotine or caffeine or had evidence for recent use of addictive drugs other than nicotine or caffeine; had a lifetime history of psychotic or bipolar disorder; or met criteria for major depressive disorder or posttraumatic stress disorder in the prior 6 months.

Screening Visit—During a screening visit, smoking and medical history, and physical examination with vital signs and weight were conducted. The Structured Clinical Interview for DSM-IV (SCID) (First et al. 1995), Hamilton Rating Scale for Depression (HAM-D) (Hamilton 1960), and Fagerstrom Test for Nicotine Dependence (FTND) (Heatherton et al. 1991) were administered. Craving was assessed with an 8-point Likert-type rating scale with the instructions "Please rate how much you feel craving for a cigarette right now" $(0=$ "no desire at all'; $7=$ 'unable to resist'). Participants with a positive screening for pregnancy (One Step HCG Urine Test, Medimpex United Inc., Bensalem, PA) or a positive urine screen for recent marijuana, cocaine, methamphetamine, amphetamine, opiate, or benzodiazepine use (Multi Drug 6 Panel Urine Test, Medimpex United Inc., Bensalem, PA) or recent ethanol use (Alco-Screen Alcohol Saliva Test Kit, Chematics North Webster IN) were excluded. Recent drug, ethanol and tobacco use (self-report and expired carbon monoxide (CO) were assessed at each visit.

\section{Visit One: Study medication administration, memory reactivation, and script} preparation-After consent and screening, participants were asked to return within one week in a 10-12 hour abstinent state by self-report, verified by expired CO $<15 \mathrm{ppm}$. Eligible participants with systolic blood pressure $>100 \mathrm{~mm} \mathrm{Hg}$ and pulse $>55 \mathrm{bpm}$ received pre-retrieval oral propranolol or identical placebo, as described previously (Brunet et al. 2011; Finnie and Nader 2012; Lonergan et al. 2013; Poundja et al. 2012). Study medication was dosed in a two-step procedure to allow for assessment of tolerability of short-acting propranolol prior to administration of the long-acting formulation. Short acting dose was $0.67 \mathrm{mg} / \mathrm{kg}$, rounded to the nearest $10 \mathrm{mg}$, minimum dose: $40 \mathrm{mg}$, maximum dose: $80 \mathrm{mg}$. Ninety minutes after the short-acting dose, participants with systolic blood pressure $>100$ $\mathrm{mm} \mathrm{Hg}$ and pulse $>50 \mathrm{bpm}$ received long-acting propranolol, $1 \mathrm{mg} / \mathrm{kg}$ rounded to the nearest $20 \mathrm{mg}$, minimum dose: $60 \mathrm{mg}$, maximum dose: $120 \mathrm{mg}$. The combination dose was expected to provide b-blockade for a 24-hour period and has been reported to block reconsolidation of traumatic memories (Brunet et al. 2008). 
Immediately following administration of long-acting study medication, participants reactivated smoking related memories by writing a detailed description of the two routine situations that most reliably trigger for them strong craving to smoke, including details of the setting, persons involved, time of day, circumstance, and description of physical sensations accompanying craving. Their descriptions were then discussed with them in detail to enhance reactivation of the most salient aspects of memories of cigarette craving. The description of the two scenarios elicited sensory and affective details in the description of wanting to smoke, and the narrative stopped before satisfaction was gained by actually smoking, eg. it focused on the time of maximal 'wanting'. An effort was made to avoid inducing extinction, insight, or reframing of memory, all of which would be undesirable outcomes given this study's rationale. The reactivation procedure used in this study was identical to that used by Brunet, Orr, et al., 2008 (Brunet et al. 2008) except that smoking craving-related memories rather than traumatic memories were elicited (see also Brunet et al. 2011; Pitman et al. 1987; Pitman et al. 2002).

From these written personal descriptions, study staff prepared two 40-second smoking scripts in the second person, present tense, incorporating as much of each subject's vernacular as possible, using a method described by Orr and colleagues, modified for use in smokers (Orr et al. 1993). Study staff also prepared two standard neutral scripts, describing situations unrelated to smoking, falling asleep and taking a shower, and created audio files by reading the scripts aloud into a microphone.

Visit 2: Assessment of Cue Reactivity-One week after visit one, participants returned in a non-deprived state for assessment of cue associated physiologic reactivity, emotional valence and craving in a one-hour, script-driven imagery session that was identical to that used by Pitman (1987) and Brunet (2008) except that smoking cravingrelated memories rather than traumatic memories were elicited. To establish baseline parameters, participants listened, through headphones, to a relaxation script, HR, SC, EMG were measured and subjects completed assessments of baseline emotional state and craving. This procedure was then repeated with two counter-balanced personalized smoking and two standard neutral scripts, $40 \mathrm{sec}$ each, beginning with a neutral script. Each script presentation consisted of four sequential 30-s periods: baseline, read, imagery, and recovery. Subjects were instructed to listen carefully during the playing of the scripts and to attempt to imagine as vividly as possible each experience as it was presented (read period) and, on script termination, to continue to imagine the experience from beginning to end (imagery period) until they heard a tone. They were further instructed to stop imagining the script at the tone and to relax (recovery period) until a second tone was heard. At this time, they made self-reports of emotion and craving on Likert-type scales. Subjects were asked to relax until their HR returned to within 5\% of baseline before listening to the next script.

Heart rate (HR), skin conductance (SC), and electromyogram (EMG) of the left corrugator facial muscle were measured with a Coulbourn Modular Instrument System. Heart rate was measured with 9-mm diameter $\mathrm{Ag} / \mathrm{AgCl}$ electrodes filled with electrolytic paste, placed on the medial forearm; HR was converted from interbeat interval. Skin conductance was assessed with 9-mm Ag/ $\mathrm{AgCl}$ electrodes filled with isotonic paste placed on the nondominant hypothenar surface with a constant-voltage technique. Corrugator EMG was 
obtained with 4-mm $\mathrm{Ag} / \mathrm{AgCl}$ electrodes. The amplified EMG signal was integrated using a 200-msec time constant. Physiologic measures were recorded during the 30-second imagery periods that followed each of the four scripts. Physiologic change scores were calculated by subtracting the mean physiologic activation level of the baseline period from the mean physiologic level of each imagery period.

In the subjective ratings of emotional state and craving participants rated their emotional state in the domains: control/dominance, pleasure, arousal, happiness, sadness, anger, fear, disgust, and guilt, using a joystick and a 13-point Likert-type rating scale presented on a computer monitor. Participants rated one scale for each emotional state with the instructions "Please rate how you felt when you were imagining the scene". Post-script craving was then assessed using an 8-point Likert-type rating scale with the instructions "Please rate how much you feel craving for a cigarette right now" $(0=$ 'no desire at all'; $7=$ 'unable to resist').

\section{Analytic Strategy}

In order to test if treatment had an effect on physiologic, emotional or craving outcomes, we fit a linear mixed effects model for each outcome (i.e., 13 dependent variables) using proc mixed in SAS (Cary, NC). In these models, predictors were randomized group assignment (propranolol vs. placebo), script type (smoking vs. neutral), time (4 observations per person), and the group*script interaction effect. We interpreted the group*script interaction effect as impact of propranolol on smoking cue reactivity, as it would demonstrate differential reactivity to smoking scripts vs. neutral scripts in those receiving propranolol vs. placebo. To account for correlation of repeated observations, we used an AR1 correlation matrix structure. To address the problem of multiple significance testing (i.e., 3 physiological and 10 self-report outcome variables), we adjusted $p$-values by controlling the expected proportion of falsely rejected hypotheses, that is, the false discovery rate (Hochberg and Benjamini 1990). The study was powered to detect a conservative but clinically meaningful between group difference in heart rate response during script-driven imagery of $15 \mathrm{bpm}$ and a conservative retention rate of $85 \%$. The power calculation using MGH Center for Biostatistics power calculator (http://hedwig.mgh.harvard.edu/ sample_size/js/js_parallel_quant.html) predicted 54 participants would need to complete this two-treatment parallel-design study to have an $85 \%$ probability of detecting a treatment difference at a two-sided 0.05 significance level, if the treatment effect is $15 \mathrm{bpm}$ with a standard deviation of $18 \mathrm{bpm}$.

\section{RESULTS}

\section{Participants}

One hundred thirteen participants enrolled, 74 were randomized to propranolol and placebo groups, and 54 completed the script-driven imagery session one week after study medication dosing. The treatment groups were comparable in age, sex, severity of nicotine dependence and demographic variables. See Table 1. There were no between group differences in baseline smoking self-report, CO, craving VAS, QSU, FTND, and WSWS between those who completed the study and those who were randomized and did not complete the study. 
Fifteen participants withdrew prematurely after receipt of study medications; 9 received propranolol, and 6 received placebo. All but 5 ( 3 on propranolol and 2 on placebo) of those who were randomized but who did not complete the study were withdrawn by study staff, primarily for medical reasons (see adverse events) and did not drop out of the protocol. To evaluate biochemical verification of self reported abstinence prior to receiving study medication at Visit 1, we applied a formula that assigns a threshold expired $\mathrm{CO}$ for each subject, accounting for the expired $\mathrm{CO}$ level measured in the afternoon, the estimated accumulation of expired $\mathrm{CO}$ during smoking the remainder of the day, and the half-life for elimination during the night (assumed to be $6 \mathrm{~h}$ ) (Rose et al. 2010). By this formula, expired $\mathrm{CO}$ is considered to meet criteria for overnight abstinence if that expired $\mathrm{CO}$ for overnight abstinence criteria $\leq 14+0.25\left(1.33 * \mathrm{CO}_{\mathrm{aft}}-14\right)$ where $\mathrm{CO}_{\mathrm{aft}}$ refers to the afternoon expired $\mathrm{CO}$ reading. All participants who went on to complete the physiology session had overnight abstinent COs that met criteria for abstinence by this formula. Though not all baseline COs for participants in this study were taken in the afternoon, we applied the formula to all participants. All participants who went on to complete the physiology session had overnight abstinent COs that met criteria for abstinence by this formula. One participant had an overnight abstinent $\mathrm{CO}$ of 15 but required a $\mathrm{CO}$ of $14.2 \mathrm{ppm}$ to qualify by the formula, but this participant did not complete the physiology session. We also completed a sub-analysis of only those participants whose $\mathrm{CO}$ level were less than or equal to $10 \mathrm{ppm}$. This analysis excluded 13 participants with a $\mathrm{CO}$ corresponding to self-report of overnight abstinence exceeded 10ppm. The results of this sub-analysis did not substantively differ from the main analysis.

\section{Cue Reactivity}

There was a main effect of script type, such that immediately after listening to scripts with personalized, smoking-craving-related content, participants had greater physiologic activation, as measured by HR, SC, EMG, higher self-reported levels of negative emotions (arousal, sadness, anger, fear, disgust, guilt), and lower self-reported levels of positive emotions (dominance, pleasure, happiness), and a higher level of self-reported craving than following neutral scripts. See Table 2, Effect of Script.

There was no main effect of study drug and no drug group by script type interaction for any of the 13 measures of cue reactivity, indicating that there was no effect of study drug on reactivity to either script type and that participants did not have differential reactivity to smoking scripts depending on their drug administration. See Table 2, Effect of Drug and Effect of Drug $\times$ Script, and Figure 1 .

\section{Adverse Events}

There were no serious adverse events. Six participants experienced transient, asymptomatic bradycardia, i.e., HR $<50$ beats per minute after taking study medication. All of the participants who experienced bradycardia were assigned to the propranolol group. All were observed in the Center until the bradycardia resolved and did not receive the second dose of study medication, long-acting propranolol. One participant developed pneumonia and required ambulatory treatment, and one participant reported feeling light-headed 2 days after 
taking study drug. Both had been assigned to placebo, and both were discontinued before the assessment of cue reactivity.

\section{DISCUSSION}

In this study, a single dose of propranolol administered immediately prior to memory reactivation had no effect on physiologic or emotional reactivity to personalized smokingrelated cues, in the form of script driven imagery, one week later. Importantly, this lack of effect was observed in an experimental design that successfully elicited cue reactivity, as indicated by physiological, emotional, and craving response to script driven imagery with smoking-related but not neutral content. The robust effect of script type was observed on every physiologic, emotional and craving outcome, indicating that the personalized smoking scripts were salient and that the cue reactivity variables chosen were sensitive measures of smoking-cue reactivity. We conclude that the model was sensitive, and propranolol, as dosed in this trial, did not reduce smoking cue reactivity.

These results contrast with reports of successful attenuation of memory reconsolidation of traumatic memories in patients with PTSD. This raises the possibility that beta-adrenergic neurotransmission functions differently with reactivation of different kinds of memories, or that declarative or emotional memories per se are less critical in driving drug craving than traumatic memories. Although immediate release propranolol given immediately following cocaine cue exposure has been reported to reduce cue elicited craving and cardiovascular reactivity 24 hours later, there was no effect on responsiveness to cocaine cues one week later (Saladin et al., 2013). These results also contrast with apparently successful memory reconsolidation blockade of response to drug cues in animal models, and demonstrate the challenge of translating an intervention that is effective in animal models to human trials. In a rodent model, a single administration of post-reactivation propranolol reduced previously established conditioned place preference (CPP) for a cocaine-paired compartment, and multiple doses eliminated previously established cocaine CPP and eliminated reinstatement (Fricks-Gleason and Marshall 2008) and alcohol reinstatement (Wouda et al. 2010). Several different compounds, acting as antagonists or agonists of muscarinic cholinergic, NMDA glutamate, and $\beta$-adrenergic receptors, administered either immediately before or after retrieval of cocaine-, amphetamine-, alcohol- or morphine-cue associations, have been found to reduce later expression of those associations in animals (Bernardi et al. 2006; Botreau et al. 2006; Kelley et al. 2007a; Kelley et al. 2007b; Milton et al. 2008a; Popik et al. 2006; Sadler et al. 2007). The positive results in these and other animal studies in which propranolol appeared to block reconsolidation of drug-related memories may be contingent upon the complexity of the drug environment and the frequency of cue exposure. In most animal studies, animals are placed in a simplistic environment with few stimuli to associate with the drug experience, whereas smokers encounter innumerable environmental cues during smoking episodes. Moreover, the animal trials employ repeated drug and cue exposure for days, whereas most smokers have experienced repeated drug and cue exposure for decades. Because the animals encounter fewer drug cues and have less frequent exposure to these cues over shorter duration than do smokers, their drug memories are likely less complex and robust than those of human smokers, and therefore memory reconsolidation may be more easily disrupted with propranolol. 
Memory reconsolidation blockade is distinct from extinction in that it can occur even when a reinforced trial is used to reactivate the memory (Bozon, 2003 \#18;Duvarci, 2004 \#17\}, however, it may not be robust to intercurrent reinforced trials during which new memories may be laid down. Studies in animal models have included daily cue exposure following propranolol dosing, but to our knowledge, no pharmacologic memory reconsolidation blockade trials in animal models included free access to drug between memory retrieval, often with a priming dose of drug following extinction, and test of cue reactivity or reinstatement. In this study, participants were asked to abstain from smoking for 10-12 hours prior to study drug administration to enhance craving during memory reactivation. Participants then smoked during the week between study drug administration and measurement of physiologic cue reactivity, in order to minimize the potentially confounding effects of nicotine withdrawal on physiologic reactivity, affect, and craving. It is possible that new smoking experiences in the week following study drug administration were associated with creation of new smoking-related memories, such that the smoking scripts may have cued these new memories during the cue reactivity session. The long-acting propranolol dose was tailored to provide only 24-hour blockade, and would therefore not be expected to interfere with the consolidation of new memories created days afterward. A trial in smokers who are abstinent between the memory reconsolidation blockade session and the cue reactivity assessment may be warranted.

A more fundamental issue that may explain the lack of effect of propranolol is that for the human addiction experience, explicit drug-related memory, while an important feature of drug use, may not be the only essential factor driving continued use. as habit learning and incentive salience tend to take on more importance with continued use. There is doubt as to whether vivid declarative memories explain the transition to addiction (Robinson and Berridge 1993). Studies have suggested that addiction involves a transition from explicit behavior, originally controlled by the memory of drug pleasure, to more automatic behavior consisting primarily of habits, which may occur without explicit cognitive expectations of a given outcome (Robbins and Everitt 1999; Tiffany 1990). Another possibility is that the driver of drug use is not the drug learning itself, but is instead the motivational impact of drug-associated cues (i.e. their ability to engage brain motivational systems). Robinson \& Berridge (1993) suggest that addictive drugs alter reward-related brain systems that mediate a basic incentive-motivational function. These neural circuits may become sensitized to specific drug effects and to drug-associated stimuli, leading to excessive attribution of incentive salience to drug-related representations. This excessive incentive salience, in turn, causes a pathological "wanting" to take drugs, and this "wanting" may be independent of drug pleasure, withdrawal, habits, or memories (Robinson and Berridge 1993; 2000). Though Robinson and Berridge do not divorce drug related associative learning processes from the development incentive sensitization, they argue that associative learning is not all there is to addiction.

\section{Limitations}

This study used a single dose of propranolol rather than a range of doses. This study also employed a single dose of propranolol rather than repeated propranolol administration, which may be associated with greater, longer-lasting effect of reconsolidation blockade on 
smoking-related memories (Fricks-Gleason and Marshall 2008). It is possible that administering study medication pre-retrieval rather than post memory reactivation in this study may have contributed to its negative results. The dose of propranolol in the current study was based on an effective dose in an experimental paradigm in which the intervention reduced physiologic reactivity to re-exposure to traumatic memories in a clinical sample with traumatic history (Brunet, et al., 2008). In this prior trial, however, propranolol was given immediately following memory retrieval, while in the present trial, propranolol was dosed immediately prior to memory retrieval. It is possible that the administration of propranolol immediately prior to retrieval may have inadvertently interfered with memory retrieval, which could, in part, explain why it failed to diminish drug-cue associations. Supporting this idea are findings that demonstrate that fully experiencing a reactivation session is necessary in order for the disruption of memory consolidation to work. For example, Milton et al. (2008) found that animals injected with propranolol, but denied a reactivation session, were unimpaired in their responding for cocaine-associated cues (Milton et al. 2008b). Similarly, Robinson and Franklin (2007) showed that propranolol only disrupted memory for morphine CPP when given post-reactivation; control animals that received propranolol in their home cage were not impaired when tested for preference $24 \mathrm{~h}$ later (Robinson and Franklin 2007). However, some have argued that that pre-retrieval propranolol interferes with retrieval (Schiller and Phelps 2011), thus possibly diminishing drug-cue associations and by that mechanism impeding memory reconsolidation blockade following retrieval. In fact, animals and humans who receive pre-retrieval propranolol still demonstrate evidence that they are able to retrieve the emotional memory as in freezing behavior in fear conditioned rodents and galvanic skin response in humans. Thus if memory is not so impaired as to interfere with retrieval, it should not be so impaired as to block reconsolidation. (Brunet et al., 2011).

The rationale for the timing of dosing chosen in this study was that dosing immediately prior to retrieval with a short acting dose of propranolol may augment efficacy on reconsolidation blockade because there would be exposure to propranolol during and immediately following retrieval without a time-lag for drug absorption. Reconsolidation is believed to begin 3-10 min after memory reactivation (Monfils et al. 2009), to take place mostly within the first $2 \mathrm{~h}$ (Przybyslawski et al. 1999) and to be over by 6 hours (Duvarci and Nader 2004), while oral propranolol reaches peak bioavailability in blood in $90 \mathrm{~min}$ (Marino et al. 1987). Since conducting their proof-of-concept study in PTSD that used post-reactivation propranolol (Brunet et al., 2008), Brunet and colleagues have used pre-reactivation propranolol in an attempt to develop what is hoped will be a more potent therapeutic protocol (see Brunet et al., 2011). Propranolol has now been reported to reduce subsequent physiologic activation when given up to 90 minutes before reactivation of traumatic memories, using the same method for reactivation and test days employed in this study, in those with PTSD and after reactivation of emotional memories in healthy adults (Finnie and Nader 2012, in a metaanalysis by Lonergan et al., 2013, in an open label trial of 6, weekly sessions of recalling traumatic events with single dose propranolol by Poundja et al., 2012, and a report of three open label trials by Brunet et al., 2011), though the methodological limitations of open-label trials may reduce confidence in these findings. 
While the physiologic measures employed appear to have been sensitive enough to detect an effect of script and thus of propranolol, our single-item VAS measure of craving may have been insufficient to capture the breadth of various dimensions of craving (Tiffany and Drobes 1991). Use of a multi-dimensional craving scale that addresses different aspects of craving may have been more sensitive to subtle effects of the drug. In addition, the use of psychophysiological measures such as heart rate, EMG, and SC, may not have been an adequate index for craving, as psychophysiological systems can serve functions that are independent of a motivation for drug use (Sayette et al. 2000). It is possible that the increases in these measures during the smoking script retrieval were due to increased anxiety or arousal, rather than only to increased craving. It is possible that the written and auditory cues used in the retrieval condition were sufficiently different from the brief narrative descriptions derived from the written/interview materials used in the test condition so as to have compromised stimulus continuity between the retrieval and test conditions, complicating interpretation of the findings. The reactivation procedure used in this study was identical to that used by Brunet, Orr, et al., 2008; Pitman et al., 1987; Pitman et al., 2002, Orr et al., 2002, and Brunet et al., 2011 except that smoking craving-related memories rather than traumatic memories were elicited. The description of the two routinely cravingrelated scenarios elicited in visit one included sensory and affective details in the description of wanting to smoke that were included in the script read at visit 2 , and the description of the scenario in the script stopped before satisfaction, eg. it focused on the time of maximal 'wanting'. While it may be possible to avoid this issue by developing scripts in a separate session and then use the same scripts in both retrieval and testing, we did not repeat the reading of the script at the reactivation and follow up visits because we wanted the stimulus to be novel, and thus maximally stimulating.

Because we did not conduct baseline assessments of cue elicited physiologic reactivity and craving, we cannot strictly rule out that the groups were not different at baseline on the primary outcome, but because the randomized groups were well balanced at baseline on measures of nicotine dependence, smoking behavior, craving, expectation that smoking will relieve negative affect, and nicotine withdrawal symptoms and because the groups were randomized and the sample size was not small, we believe that it is unlikely that the groups were systematically different on cue reactivity at baseline. Participants in this study were heterogeneous in both age and smoking history. Finally, the integrity of the blind was not formally assessed, which may have influenced the results via participant expectancy or lack of expectancy about the effects of the drug on craving. These factors may have influenced differences in memory consolidation among these individuals, which, in turn, may have affected any overall effect of propranolol.

\section{Conclusion}

In summary, single dose, pre-retrieval propranolol after overnight smoking abstinence did not attenuate the effect of personalized, smoking-related, script-driven imagery to increase physiologic activation, craving and negative emotional reactivity in non-deprived smokers. We conclude that nicotine and other components of tobacco smoke may have pharmacologic effects on cue reactivity that work via pathways independent of the site of action of propranolol. However, memory reconsolidation blockade has shown promise in animal 
models of drug dependence (e.g. (Przybyslawski et al. 1999), indicating that more work may be needed to translate the animal model of drug dependence to a therapeutic intervention that can be tested the clinic. It may be premature to rule out an inhibitory role for propranolol in reconsolidation blockade of smoking-related memories until trials are done in recently abstinent smokers, with a range of doses (eg. Poundja et al., 2013), and with repeated propranolol administration in order to detect whether a greater, longer-lasting effect of reconsolidation blockade on smoking-related memories can be obtained.

\section{Acknowledgments}

We would like to thank the staff of the MGH PTSD physiology laboratory for their assistance in this study.

Funding

Funded by NIDA R21 DA025186, Memory Reconsolidation Blockade as a Novel Intervention for Nicotine Dependence (Evins), K24 DA030443, Mentoring in Addiction Research (Evins), R25DA026401, K01 DA DA034093 (Gilman) and K01 DA02709 (Hoeppner).

\section{REFERENCES}

Abrams DB, Monti PM, Carey KB, Pinto RP, Jacobus SI. Reactivity to smoking cues and relapse: two studies of discriminant validity. Behav Res Ther. 1988; 26:225-233. [PubMed: 3408457]

Abrams DB, Monti PM, Pinto RP, Elder JP, Brown RA, Jacobus SI. Psychosocial stress and coping in smokers who relapse or quit. Health Psychol. 1987; 6:289-303. [PubMed: 3301320]

Alberini CM. Mechanisms of memory stabilization: are consolidation and reconsolidation similar or distinct processes? Trends Neurosci. 2005; 28:51-56. [PubMed: 15626497]

Auber A, Tedesco V, Jones CE, Monfils MH, Chiamulera C. Post-retrieval extinction as reconsolidation interference: methodological issues or boundary conditions? Psychopharmacology. 2013; 226:631-647. [PubMed: 23404065]

Bernardi RE, Lattal KM, Berger SP. Postretrieval propranolol disrupts a cocaine conditioned place preference. Neuroreport. 2006; 17:1443-1447. [PubMed: 16932155]

Botreau F, Paolone G, Stewart J. d-Cycloserine facilitates extinction of a cocaine-induced conditioned place preference. Behavioural brain research. 2006; 172:173-178. [PubMed: 16769132]

Bozon B, Davis S, Laroche S. A requirement for the immediate early gene zif268 in reconsolidation of recognition memory after retrieval. Neuron. 2003; 40:695-701. [PubMed: 14622575]

Brunet A, Orr SP, Tremblay J, Robertson K, Nader K, Pitman RK. Effect of post-retrieval propranolol on psychophysiologic responding during subsequent script-driven traumatic imagery in posttraumatic stress disorder. J Psychiatr Res. 2008; 42:503-506. [PubMed: 17588604]

Brunet A, Poundja J, Tremblay J, Bui E, Thomas E, Orr SP, Azzoug A, Birmes P, Pitman RK. Trauma reactivation under the influence of propranolol decreases posttraumatic stress symptoms and disorder: 3 open-label trials. J Clin Psychopharmacol. 2011; 31:547-550. [PubMed: 21720237]

Chiamulera C, Costa S, Reggiani A. Effect of NMDA- and strychnine-insensitive glycine site antagonists on NMDA-mediated convulsions and learning. Psychopharmacology. 1990; 102:551552. [PubMed: 1982904]

Childress AR, McLellan AT, Ehrman R, O'Brien CP. Classically conditioned responses in opioid and cocaine dependence: a role in relapse? NIDA Res Monogr. 1988; 84:25-43. [PubMed: 3147384]

Conklin CA, Tiffany ST. The impact of imagining personalized versus standardized urge scenarios on cigarette craving and autonomic reactivity. Experimental and Clinical Psychopharmacology. 2001; 9:399-408. [PubMed: 11764016]

Debiec J, Ledoux JE. Disruption of reconsolidation but not consolidation of auditory fear conditioning by noradrenergic blockade in the amygdala. Neuroscience. 2004; 129:267-272. [PubMed: 15501585] 
Diergaarde L, Schoffelmeer AN, De Vries TJ. Beta-adrenoceptor mediated inhibition of long-term reward-related memory reconsolidation. Behav Brain Res. 2006; 170:333-336. [PubMed: 16600394]

Drobes DJ, Tiffany ST. Induction of smoking urge through imaginal and in vivo procedures: physiological and self-report manifestations. J Abnorm Psychol. 1997; 106:15-25. [PubMed: 9103714]

Duvarci S, Nader K. Characterization of fear memory reconsolidation. J Neurosci. 2004; 24:9269_ 9275. [PubMed: 15496662]

Finnie PS, Nader K. The role of metaplasticity mechanisms in regulating memory destabilization and reconsolidation. Neurosci Biobehav Rev. 2012; 36:1667-1707. [PubMed: 22484475]

First, MB.; Spitzer, RL.; Gibbon, M.; Williams, JBW. Structured Clinical Interview for DSM-IV Axis I Disorders--Patient Edition (SCID-I/P, Version 2.0). New York: Biometrics Research Department, New York State Psychiatric Institute; 1995.

Fricks-Gleason AN, Marshall JF. Post-retrieval beta-adrenergic receptor blockade: effects on extinction and reconsolidation of cocaine-cue memories. Learn Mem. 2008; 15:643-648. [PubMed: 18772251]

Grant S, London ED, Newlin DB, Villemagne VL, Liu X, Contoreggi C, Phillips RL, Kimes AS, Margolin A. Activation of memory circuits during cue-elicited cocaine craving. Proc Natl Acad Sci U S A. 1996; 93:12040-12045. [PubMed: 8876259]

Hamilton M. A rating scale for depression. J Neurol Neurosurg Psychiatry. 1960; 23:56-62. [PubMed: 14399272]

Heatherton TF, Kozlowski LT, Frecker RC, Fagerstrom KO. The Fagerstrom test for nicotine dependence: a revision of the Fagerstrom tolerance questionnaire. Brit J Addict. 1991; 86:11191127. [PubMed: 1932883]

Hochberg Y, Benjamini Y. More powerful procedures for multiple significance testing. Stat Med. 1990; 9:811-818. [PubMed: 2218183]

Kelley BJ, Yeager KR, Pepper TH, Bornstein RA, Beversdorf DQ. The effect of propranolol on cognitive flexibility and memory in acute cocaine withdrawal. Neurocase. 2007a; 13:320-327. [PubMed: 18781429]

Kelley JB, Anderson KL, Itzhak Y. Long-term memory of cocaine-associated context: disruption and reinstatement. Neuroreport. 2007b; 18:777-780. [PubMed: 17471065]

Kindt M, Soeter M, Vervliet B. Beyond extinction: erasing human fear responses and preventing the return of fear. Nat Neurosci. 2009; 12:256-258. [PubMed: 19219038]

Lee JL, Di Ciano P, Thomas KL, Everitt BJ. Disrupting reconsolidation of drug memories reduces cocaine-seeking behavior. Neuron. 2005; 47:795-801. [PubMed: 16157275]

Lonergan MH, Olivera-Figueroa LA, Pitman RK, Brunet A. Propranolol's effects on the consolidation and reconsolidation of long-term emotional memory in healthy participants: a meta-analysis. $\mathrm{J}$ Psychiatry Neurosci. 2013; 38:222-231. [PubMed: 23182304]

Marino MR, Dey M, Garg DC, Jallad NS, Dorick DM, Martinez JJ, Weidler DJ. Pharmacokinetics and pharmacodynamics of long-acting propranolol 60-mg capsules: a comparative evaluation. J Clin Pharmacol. 1987; 27:885-891. [PubMed: 3429696]

Milton AL, Lee JL, Butler VJ, Gardner R, Everitt BJ. Intra-amygdala and systemic antagonism of NMDA receptors prevents the reconsolidation of drug-associated memory and impairs subsequently both novel and previously acquired drug-seeking behaviors. The Journal of neuroscience : the official journal of the Society for Neuroscience. 2008a; 28:8230-8237. [PubMed: 18701685]

Milton AL, Lee JL, Everitt BJ. Reconsolidation of appetitive memories for both natural and drug reinforcement is dependent on \{beta\}-adrenergic receptors. Learning \& memory. 2008b; 15:8892. [PubMed: 18235109]

Monfils MH, Cowansage KK, Klann E, LeDoux JE. Extinction-reconsolidation boundaries: key to persistent attenuation of fear memories. Science. 2009; 324:951-955. [PubMed: 19342552]

Nader K. Neuroscience: re-recording human memories. Nature. 2003; 425:571-572. [PubMed: 14534572] 
Nader K, Schafe GE, Le Doux JE. Fear memories require protein synthesis in the amygdala for reconsolidation after retrieval. Nature. 2000; 406:722-726. [PubMed: 10963596]

Orr SP, Pitman RK, Lasko NB, Herz LR. Psychophysiological assessment of posttraumatic stress disorder imagery in World War II and Korean combat veterans. J Abnorm Psychol. 1993; 102:152-159. [PubMed: 8436691]

Pitman RK, Orr SP, Forgue DF, de Jong JB, Claiborn JM. Psychophysiologic assessment of posttraumatic stress disorder imagery in Vietnam combat veterans. Arch Gen Psychiatry. 1987; 44:970-975. [PubMed: 3675137]

Pitman RK, Sanders KM, Zusman RM, Healy AR, Cheema F, Lasko NB, Cahill L, Orr SP. Pilot study of secondary prevention of posttraumatic stress disorder with propranolol. Biol Psychiatry. 2002; 51:189-192. [PubMed: 11822998]

Popik P, Wrobel M, Bisaga A. Reinstatement of morphine-conditioned reward is blocked by memantine. Neuropsychopharmacology. 2006; 31:160-170. [PubMed: 15886718]

Poundja J, Sanche S, Tremblay J, Brunet A. Trauma reactivation under the influence of propranolol: an examination of clinical predictors. Eur J Psychotraumatol. 2012; 3

Przybyslawski J, Roullet P, Sara SJ. Attenuation of emotional and nonemotional memories after their reactivation: role of beta adrenergic receptors. J Neurosci. 1999; 19:6623-6628. [PubMed: 10414990]

Rickard-Figueroa K, Zeichner A. Assessment of smoking urge and its concomitants under an environmental smoking cue manipulation. Addict Behav. 1985; 10:249-256. [PubMed: 4083102]

Robbins TW, Everitt BJ. Drug addiction: bad habits add up. Nature. 1999; 398:567-570. [PubMed: 10217139]

Robinson MJ, Franklin KB. Central but not peripheral beta-adrenergic antagonism blocks reconsolidation for a morphine place preference. Behav Brain Res. 2007; 182:129-134. [PubMed: 17604134]

Robinson TE, Berridge KC. The neural basis of drug craving: an incentive-sensitization theory of addiction. Brain Res Brain Res Rev. 1993; 18:247-291. [PubMed: 8401595]

Robinson TE, Berridge KC. The psychology and neurobiology of addiction: an incentive-sensitization view. Addiction. 2000; 95(Suppl 2):S91-S117. [PubMed: 11002906]

Rose JE, Salley A, Behm FM, Bates JE, Westman EC. Reinforcing effects of nicotine and non-nicotine components of cigarette smoke. Psychopharmacology. 2010; 210:1-12. [PubMed: 20358364]

Sadler R, Herzig V, Schmidt WJ. Repeated treatment with the NMDA antagonist MK-801 disrupts reconsolidation of memory for amphetamine-conditioned place preference. Behav Pharmacol. 2007; 18:699-703. [PubMed: 17912055]

Sayette MA, Shiffman S, Tiffany ST, Niaura RS, Martin CS, Shadel WG. The measurement of drug craving. Addiction. 2000; 95(Suppl 2):S189-S210. [PubMed: 11002914]

Shiffman S. Dynamic influences on smoking relapse process. J Pers. 2005; 73:1715-1748. [PubMed: 16274451]

Suzuki A, Josselyn SA, Frankland PW, Masushige S, Silva AJ, Kida S. Memory reconsolidation and extinction have distinct temporal and biochemical signatures. J Neurosci. 2004; 24:4787-4795. [PubMed: 15152039]

Tiffany ST. A cognitive model of drug urges and drug-use behavior: role of automatic and nonautomatic processes. Psychol Rev. 1990; 97:147-168. [PubMed: 2186423]

Tiffany ST, Drobes DJ. The development and initial validation of a questionnaire on smoking urges. Br J Addict. 1991; 86:1467-1476. [PubMed: 1777741]

Tiffany ST, Hakenewerth DM. The production of smoking urges through an imagery manipulation: psychophysiological and verbal manifestations. Addict Behav. 1991; 16:389-400. [PubMed: 1801563]

Weiss F. Neurobiology of craving, conditioned reward and relapse. Curr Opin Pharmacol. 2005; 5:919. [PubMed: 15661620]

Wouda JA, Diergaarde L, Riga D, van Mourik Y, Schoffelmeer AN, De Vries TJ. Disruption of LongTerm Alcohol-Related Memory Reconsolidation: Role of beta-Adrenoceptors and NMDA Receptors. Front Behav Neurosci. 2010; 4:179. [PubMed: 21152256] 
Xue YX, Luo YX, Wu P, Shi HS, Xue LF, Chen C, Zhu WL, Ding ZB, Bao YP, Shi J, Epstein DH, Shaham Y, Lu L. A memory retrieval-extinction procedure to prevent drug craving and relapse. Science. 2012; 336:241-245. [PubMed: 22499948]

Zhao LY, Sun LL, Shi J, Li P, Zhang Y, Lu L. Effects of beta-adrenergic receptor blockade on drugrelated memory reconsolidation in abstinent heroin addicts. Drug Alcohol Depend. 2011; 118:224-229. [PubMed: 21531091]

Zhao LY, Zhang XL, Shi J, Epstein DH, Lu L. Psychosocial stress after reactivation of drugrelated memory impairs later recall in abstinent heroin addicts. Psychopharmacology. 2009; 203:599-608. [PubMed: 19020867] 


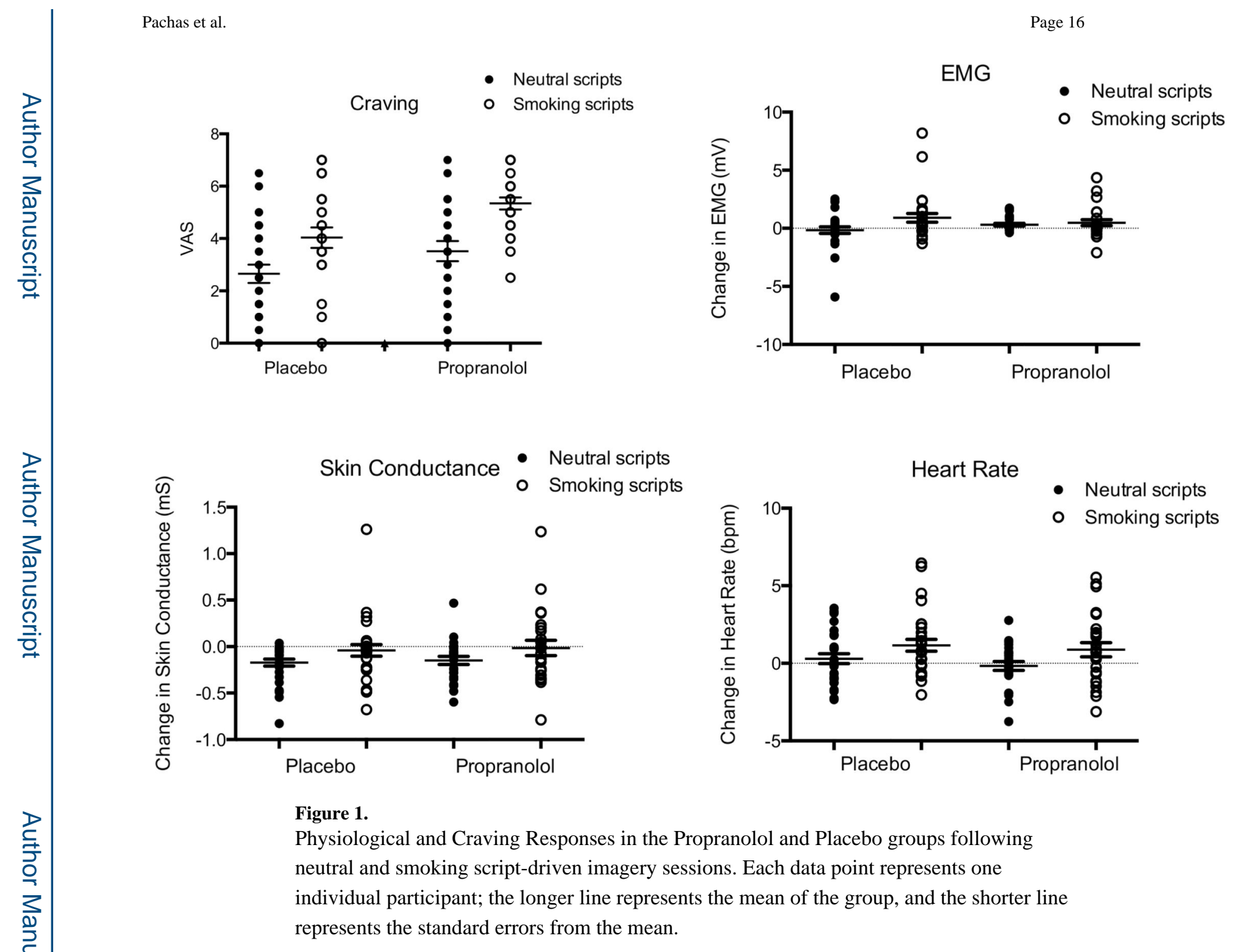

Psychopharmacology (Berl). Author manuscript; available in PMC 2016 May 01. 


\section{Table 1}

Participant Characteristics

\begin{tabular}{lccc}
\hline & $\begin{array}{c}\text { Propranolol } \\
(\mathbf{n}=\mathbf{3 5})\end{array}$ & $\begin{array}{c}\text { Placebo } \\
(\mathbf{n = 3 9 )}\end{array}$ & $\boldsymbol{p}$ \\
\hline Male Sex & $27(77 \%)$ & $27(69 \%)$ & 0.44 \\
Age (years) & $41.6(10.9)$ & $42.5(9.8)$ & 0.70 \\
More than 12 yrs education & $19(60 \%)$ & $21(54 \%)$ & 0.75 \\
Age began daily smoking & $17.5(5.2)$ & $16.5(3.2)$ & 0.29 \\
Years of regular smoking & $20.5(12.2)$ & $24.0(10.4)$ & 0.18 \\
Cigarettes/day in the 7 days prior to enrollment & $18.4(5.7)$ & $17.3(6.6)$ & 0.44 \\
Cigarettes per day since initiation & $18.3(7.4)$ & $16.5(5.3)$ & 0.29 \\
FTND total score & $5.4(2.0)$ & $5.6(1.9)$ & 0.66 \\
QSU total score & $29.7(9.3)$ & $27.0(11.2)$ & 0.34 \\
WSWS total score & $52.1(16.0)$ & $50.7(18.3)$ & 0.77 \\
Self reported craving at visit 1 (Visual analogue scale) & $4.7(1.4)$ & $4.1(1.6)$ & 0.13 \\
CO at screening (ppm) & $13.1(7.5)$ & $14.3(5.5)$ & 0.44 \\
CO at randomization (ppm) & $6.6(3.4)$ & $7.6(3.8)$ & 0.27 \\
CO at physiology session (ppm) & $18.1(10.7)$ & $15.5(6.2)$ & 0.27 \\
\hline
\end{tabular}

Values in parenthesis are standard deviations.

FTND $=$ Fagerstrom Test for Nicotine Dependence, assesses severity of physiological dependence on nicotine, scores greater than or equal to 5 indicate severe nicotine dependence

QSU = Questionnaire of Smoking Urges

WSWS =Wisconsin Smoking Withdrawal Scale

$\mathrm{CO}=$ carbon monoxide

ppm $=$ parts per million 
